\title{
BIO.08 - Improving the autocleavage performance of human L-asparaginase: a novel solution to overcome the challenges of leukemia treatment
}

\author{
Stephanie Bath de Morais ${ }^{1 *}$; Tatiana de Arruda Campos Brasil de Souza ${ }^{1}$.
}

1Fiocruz - Paraná.

Introduction: Besides its serious side effects, bacterial L-asparaginases have been a vital component of acute lymphoblastic leukemia therapy for over 40 years. Treatment efficiency relies on ability of bacterial L-asparaginases in depleting plasma asparagine, that are essential for tumor progression. Nowadays, three asparaginases are used in therapy: native and a PEGylated form of L-asparaginase II from Escherichia coli, and L-asparaginase from Erwinia chrysanthemi. The E. coli L-asparaginase can lead to clinical hypersensitivity reactions in up to $60 \%$ of patients and/or trigger several toxic reactions. Although the use of Erwinia chrysanthemi L-asparaginase is associated with lower occurrence of side effects, this enzyme is less stable, which increases injections frequency. The great progress of therapeutic use of human L-asparaginase would be to decrease toxicity and immunogenicity; however human L-asparaginase needs to undergo activation through an autocleavage step, which was shown to be a low efficiency process in vitro, reducing enzyme activity and hindering its therapeutic use.

Objective: Producing an engineered human L-asparaginase with improved ability of in vitro autocleavage

Methodology: Structural analysis of human L-asparaginases structures (PDB 4O0C, 4OSX and 4OSY) investigated the determinants of autocleavage step. In silico approaches simulated potentiality of proposed mutants. Wild-type and modified enzymes were cloned, expressed as recombinant and purified by chromatographic methods. SDS-PAGE and western blot assay detected cleaved enzymes. Biochemical assays measured L-asparaginase activity. Those experimental procedure together with in vivo assays comprise pre-clinical studies that will provide the chemotherapeutic efficiency.

Results: Structure and in silico analysis indicated that the modification of residues comprising the conserved glycine-rich loop seemed advantageous. SDS-PAGE and western blot confirmed the higher rate of cleavage in the glycine-rich loop mutant enzyme. Mutant presented catalytic efficiency 40 times higher $\left(\mathrm{k}_{\text {cat }} 21.9 \pm 0.6^{\mathrm{s}-1}\right)$ in comparison with wild-type enzyme $\left(\mathrm{k}_{\text {cat }}\right.$ $\left.0.5 \pm 0.2^{\mathrm{s}-1}\right)$. We observed for the first-time significantly high rates of human L-asparaginase autoprocessing and L-asparaginase activity in vitro without external accelerators once all information for activation is contained in the mutant protein sequence. This original invention is deposited (BR1020180010336). Mutant human L-asparaginase still presents lower activity than the first choice L-asparaginase in leukemia treatment (E. coli $\mathrm{L}$-asparaginase, $\mathrm{k}_{\text {cat }}$ 49,35 ${ }^{\mathrm{s}-1}$ ). However, this clinical disadvantage might be overcome by enhancing the dose with human enzyme. The non-immunogenic behavior of human L-asparaginase together with its high specificity to asparagine and absence of glutaminase activity allowed higher doses in treatment without the toxic and hypersensitivity effects observed with non-human L-asparaginases.

Conclusion: This work presented the production of engineered human L-asparaginase with improved autocleavage and higher L-asparaginase activity, which is novel and would allow deeper mechanistic understanding of autocleavage step. The use of human L-asparaginase in leukemia treatment has several clinical advantages, wherein the production of a human L-asparaginase with improved autocleavage is the first step to make it true.

Keywords: L-asparaginase; acute lymphoblastic leukemia; protein engineering 